\title{
INTERNAL FRICTION IN F.C.C. ALLOYS DUE TO SOLUTE DRAG ON DISLOCATIONS-I. A MODEL FOR THE EFFECT OF CORE DIFFUSION
}

\author{
A. PICHLER $\dagger$ and E. ARZT \\ Max-Planck-Institut für Metallforschung and Institut für Metallkunde der Universität Stuttgart, \\ D-70174 Stuttgart, Germany
}

(Received 16 July 1993; in revised form 23 February 1994)

\begin{abstract}
The internal friction (mechanical loss) behavior of dislocations is studied in a model which, for the first time, considers the substitutional solute mobility in the dislocation core to be higher than in the bulk around it. The parameters investigated include the external stress $\sigma_{x y}$, the solute concentration $c_{0}$, the pinning length of the dislocation and the temperature. It is shown that, at low $c_{0}$ and high $\sigma_{x y}$, the kinetics of the dislocation motion is determined by the fast diffusion of the solute atoms in the core, while for high $c_{0}$ and low $\sigma_{x y}$ the diffusion of the atoms far away from the dislocation is rate-limiting. The results are compared with the analytical model of Schoeck and are applied to the alloy system $\mathrm{Al}-\mathrm{Si}$. New experimental results supporting the model are described in a companion paper (Part II).
\end{abstract}

\section{INTRODUCTION}

Dislocations are of great importance in the interpretation of many internal friction (mechanical loss) experiments. The kinetics of the dislocation movement may be controlled by different mechanisms [1-4]. Generally, intrinsic dislocation mechanisms, such as the non-conservative movement of jogs on screw dislocations [5], can be distinguished from extrinsic mechanisms, which involve the interaction with other defects, such as solute atoms [6-8]. For describing the movement of a dislocation in internal friction experiments two different model levels are common: The first is based on the KoehlerGranato-Lücke $[9,10]$ string model, which considers only the line tension and the stress field of a dislocation. On the other level, also the atomistic structure, the periodicity of the lattice and the Peierls potential are taken into account [2]. In materials which are not very pure the following effects can occur:

(i) breakaway from the solute atom cloud $[8,11,12]$

(ii) concurrent motion of dislocation and solute atom cloud $[2,6,13]$.

Which case dominates is determined by material parameters (e.g. solute atom concentration) and the testing parameters external stress and temperature.

In this paper only the parameter range in which the dislocation cannot move without the solute atoms is investigated. For this case, longitudinal movement of

$\ddagger$ Present address: VOEST ALPINE Stahl Linz GmbH, Linz, Austria. the solute, i.e. along the dislocation, and transverse movement, i.e. normal to the dislocation, are considered $[13,14]$. The motivation for this study originated in some internal friction results for $\mathrm{Al}-\mathrm{Si}$ single crystals $[15,16]$, where a loss maximum was obtained which was caused by dislocations but had a lower activation enthalpy than that for volume diffusion. As longitudinal movement and breakaway from the solute cloud could be excluded, this behavior was in principle explained by a transverse movement of the solute atoms and by the main assumption of a higher mobility (lower activation enthalpy) for solute diffusion in the dislocation core. It remained unclear however under which conditions these solute atoms in the core region determine the activation enthalpy of the internal friction process.

While a higher longitudinal mobility of the solute in the dislocation core is well established for internal friction $[4,13,17]$, the possibility of a lower activation enthalpy for transverse diffusion in the dislocation core has not been considered in the literature up to now. The following numerical model studies the consequences of such an effect as a function of the parameters external shear stress $\sigma_{x y}$, solute atom concentration $c_{0}$, length $l$ of the dislocation between the pinning points and temperature $T$. The calculations are conducted for the quasistatic case, in which a constant stress $\sigma_{x y}$ acts on the dislocation. The bowing of the dislocation and the resulting anelastic strain are computed as a function of time. From this response function, the strain as a function of time and the relaxation parameter for dynamic internal friction are deduced. The symbols used are listed in the Nomenclature at the end. 


\section{THE MODEL}

\subsection{Assumptions and approximations}

Consider an edge dislocation in a material, pinned at distances $l$ by dislocation nodes or particles and surrounded by a Cottrell cloud of substitutional solute atoms. An external stress now attempts to bow out the dislocation between the pinning points. Only the case in which the concurrent movement of the Cottrell cloud determines the kinetics is considered. Dislocation movement stops once the force on the dislocation is balanced by the line tension.

In order to arrive at a tractable model the following approximations are made:

(i) The dislocation is treated as a flexible string [3, 18]. This is admissible if the deviation between the Peierls valley and the dislocation line is large enough (for f.c.c. metals larger than $\sim 4^{\circ}$ [1]) for the kinks to overlap. The line tension $T_{\mathrm{L}}$ is given to a reasonable approximation by $G b^{2} / 2$, where $G$ is the shear modulus and $b$ the Burgers vector. The discrete nature of the lattice and of the Peierls relief are not considered. Therefore a contribution from the kink pair generation to the activation energy $(\sim 0.1 \mathrm{eV}$ for $\mathrm{Al}$ ) is neglected for dislocations parallel to the Peierls valley; this important local mechanism should not greatly influence the overall behavior of the solute cloud.

(ii) The dislocation field is described by the elastic field of a Peierls-Nabarro-dislocation [19], an assumption which avoids the stress singularity at the dislocation core.

(iii) During bowing of the dislocation, the elements $\mathrm{d} s$ along the line (Fig. 1) have different velocities. To circumvent a time-consuming threedimensional problem, a rigid dislocation with a constant velocity is assumed. The influence of the bowing-out is simulated by subtracting from the external stress a back stress of the following form (for derivation see Appendix Al)

$$
\sigma_{\mathrm{L}}=4 \frac{G b}{l^{2}} x_{\mathrm{d}}
$$

where $x_{\mathrm{d}}$ is the bowing-out distance of the dislocation between the pinning points.

(iv) The distribution of the solute atoms around the dislocation is described by a concentration field

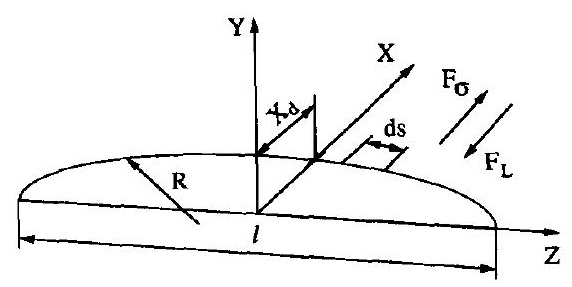

Fig. 1. Configuration for describing the movement of a pinned edge dislocation under an external stress $\sigma_{x y} . F_{\sigma}$ denotes the force due to the external stress and $F_{L}$ the force due to the back stress arising from the line tension. $c=c(x, y)$. This function can be interpreted as the probability of finding a solute atom in an infinitesimal volume element. The movement and the effects of single atoms are not considered in the model.

(v) The field around the dislocation is divided into two regions (Fig. 2): core diffusion is assumed to dominate in a square interior region with an edge length of $2 b$ ("core region"), outside this region only bulk diffusion operates ("bulk region"). Diffusion of the solute atoms is described by a jump rate equation.

\subsection{Model calculations}

The calculation procedure can be summarized as follows. First, a finite-difference mesh is constructed around the dislocation as shown in Fig. 2. (The nodes of this mesh are of course not atomic positions.) Then the equilibrium distribution of a given concentration of solute atoms in the vicinity of the dislocation is calculated and the corresponding values are allocated to the nodes. At time $t=0$ an external stress is applied, which bows the dislocation until the forces due to external stress, line tension and solute atoms balance to zero. Next the solute cloud, which is now not in equilibrium with the dislocation, has to be adjusted: in a time step $\Delta t$ the solute atoms move by diffusion toward a new equilibrium distribution. After this diffusion step the force exerted on the dislocation by the solute atoms is lower and the dislocation can bow further. By a combination of these two steps the bowing of the dislocation is calculated as a function of time. The dislocation motion finally stops when the force due to the line tension is equal to the force due to the external stress; the solute atoms, now in equilibrium, do no longer exert any stress.

For the initial solute distribution around the straight dislocation an ideal segregation obeying the Fermi-Dirac distribution [20] is assumed

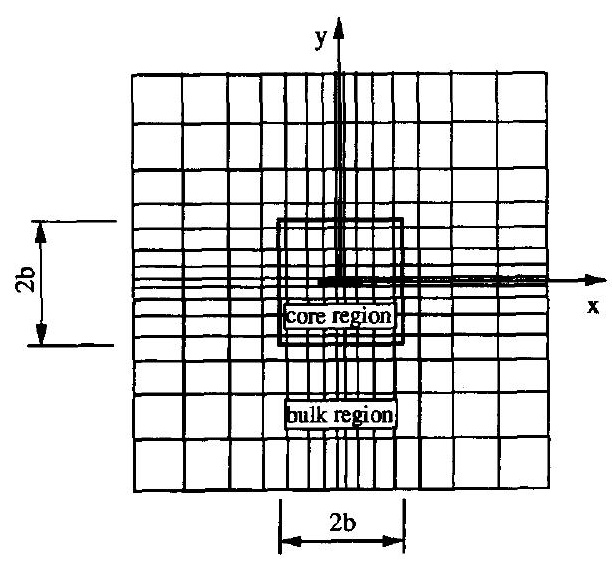

Fig. 2. Schematic of the mesh for solving the diffusion problem and for calculating the forces exerted on the dislocation by the solute atoms. For the core and bulk regions different solute diffusivities are assumed. 


$$
c(x, y)=\frac{1}{1-\frac{1-c_{0}}{c_{0}} \cdot \exp (E / k T)}
$$

where $c_{0}$ is the number concentration far away from the dislocation, $E$ the interaction energy between dislocation and solute atom, $k$ Boltzmann's constant, and $T$ the absolute temperature.

For this interaction energy the elastic solution for a misfitting sphere is used [21]

$$
E=A \frac{y \pm \xi}{\left(x-x_{\mathrm{d}}\right)^{2}+(y \pm \xi)^{2}}
$$

where

$$
A=\frac{1+v}{1-v} \cdot \frac{G \cdot b}{\pi} \cdot \epsilon \cdot \Omega
$$

and $\Omega$ is the atomic volume of the matrix, $\epsilon$ the elastic misfit of a solute atom, $v$ Poisson's ratio, and $\xi$ the dislocation half width. In equation (3) the + sign is used for $y \geqslant 0$ and the - sign for $y<0$. A computation for $\mathrm{Si}$ in $\mathrm{Al}$ with the material parameters in Table 1 gives the realistic energy of $0.23 \mathrm{eV}$ for the interaction between a solute atom in the dislocation core and the dislocation.

The force exerted by a misfitting solute atom at $x, y$ on a dislocation bowed out to $x_{\mathrm{d}}$ is calculated according to

$$
F=-\frac{\partial E}{\partial x_{\mathrm{d}}}=2 A \cdot \frac{\left(x_{\mathrm{d}}-x\right) \cdot(y \pm \xi)}{\left[\left(x-x_{\mathrm{d}}\right)^{2}+(y \pm \xi)^{2}\right]^{2}}
$$

For computing the force due to the whole Cottrell cloud, equation (4) is multiplied by the probability $c$ of finding a solute atom at $x, y$ in the volume $\mathrm{d} V$ and integrated over the volume. Because of the straight dislocation assumption we integrate over the plane $x, y$ to get the force per unit length along $z$

$$
\begin{array}{r}
F_{\mathrm{s}}=2 A \int_{-\infty}^{+\infty} \int_{-\infty}^{+\infty} \frac{\left(x_{\mathrm{d}}-x\right) \cdot(y \pm \xi)}{\left[\left(x-x_{\mathrm{d}}\right)^{2}+(y \pm \xi)^{2}\right]^{2}} \\
\times \frac{c(x, y)}{\Omega} \cdot \mathrm{d} x \cdot \mathrm{d} y .
\end{array}
$$

For computing the force it is immaterial whether the dislocation moves by $x_{\mathrm{d}}$ in the Cottrell cloud or the Cottrell cloud moves by $x_{\mathrm{d}}$ in the opposite direction. For computational simplicity, the dislocation position is fixed and the solute cloud is moved.

Finally, to readjust the Cottrell cloud, a jump rate equation of the following form must be solved [22, 23]

Table 1. Material parameters used in the calculations, with

\begin{tabular}{ccc} 
& references & \\
\hline$\epsilon_{\mathrm{Si} \text { in Al }}$ & -0.045 & {$[25]$} \\
$b(\mathrm{~nm})$ & 0.286 & {$[21]$} \\
$\xi(\mathrm{nm})$ & 0.1 & {$[21]$} \\
$G(\mathrm{GPa})$ & 26.5 & {$[21]$} \\
$\nu$ & 0.347 & {$[21]$} \\
$\Omega\left(\mathrm{m}^{3}\right)$ & $1.66 \times 10^{-29}$ & {$[21]$} \\
$D_{\mathbf{B}}\left(\mathrm{m}^{2} \cdot \mathrm{s}^{-1}\right)$ & $1.7 \times 10^{-4} \cdot \exp (-142,000 / R T)$ & {$[26]$} \\
$D_{\mathrm{C}}\left(\mathrm{m}^{2} \cdot \mathrm{s}^{-1}\right)$ & $1.7 \times 10^{-4} \cdot \exp (-82,000 / R T)$ & {$[26]$} \\
\hline
\end{tabular}

$$
\begin{aligned}
\frac{\Delta c_{i, j}}{\Delta t} & =\frac{D}{h^{2}} \cdot\left[c_{i, j}\left(1-c_{i, j-1}\right) \cdot \exp \left(\frac{E_{i, j}-E_{i, j-1}}{2 k T}\right)\right. \\
& \left.-c_{i, j-1}\left(1-c_{i, j}\right) \cdot \exp \left(\frac{E_{i, j-1}-E_{i, j}}{2 k T}\right)+\cdots\right]
\end{aligned}
$$

where $c_{i, j}$ is the concentration at node $(i, j), E_{i, j}$ the potential energy and $\Delta c_{i, j} / \Delta t$ the change in concentration in a time step, and $h$ is the distance between adjacent nodes. The appropriate diffusion coefficient $D$ is selected by insertion of the corresponding value of $Q$.

After each diffusion step a new equilibrium position $x_{\mathrm{d}}$, at which the sum of the forces $F_{\mathrm{S}}+F_{\sigma}-F_{\mathrm{L}}$ is zero, has to calculated, where $F_{\sigma}=\sigma_{x y} \cdot b$ is the external force per unit length. Therefore the implicit equation

$$
\begin{aligned}
& \sigma_{x y} \cdot b-4 \cdot \frac{G \cdot b^{2}}{l^{2}} \cdot x_{\mathrm{d}}+2 A \int_{-\infty}^{+\infty} \int_{-\infty}^{+\infty} \\
& \times \frac{\left(x_{\mathrm{d}}-x\right) \cdot(y \pm \xi)}{\left[\left(x-x_{\mathrm{d}}\right)^{2}+(y \pm \xi)^{2}\right]^{2}} \cdot \frac{c(x, y)}{\Omega} \cdot \mathrm{d} x \cdot \mathrm{d} y=0
\end{aligned}
$$

has to be solved. The equilibrium value $x_{\mathrm{d}}$ is calculated by regula falsi. To get a sufficiently exact solution for equations (6) and (7), a finer mesh is chosen in the core region than in the bulk region; this is achieved by transforming the problem to a $u, v$ space according to James and Barnett [24].

In order to extract relaxation times $\tau$ and relaxation strengths $\Delta$ from the calculated data, the bowing-out distance as a function of time is fitted by

$$
x_{\mathrm{d}}=\bar{x}_{\mathrm{d}} \cdot[1-\exp (-t / \tau)]
$$

where $\bar{x}_{\mathrm{d}}$ is the saturation value of the bowing-out distance. As will be seen, the bowing-out distance often saturates below the maximum possible value because of the line tension. It is therefore useful to define a normalized relaxation strength

$$
\Delta=\frac{\bar{x}_{\mathrm{d}}}{x_{\mathrm{d}, \max }}
$$

where $x_{\mathrm{d}, \max }$ is the maximum bowing-out distance limited by the line tension.

\section{RESULTS}

In this paper all relaxations were calculated for Si solute atoms in an Al matrix (see data in Table 1). As diffusion constants for $\mathrm{Si}$ in $\mathrm{Al}$, especially for diffusion in the core, are not known, the values for Al selfdiffusion were used. The "standard parameters", which were selected for all calculations (unless stated otherwise), were $T=400 \mathrm{~K}, l=$ $500 \mathrm{~nm}$, and $\sigma_{x y}=10^{-5} \cdot G$.

\subsection{A computing example and its evaluation}

In the following example the consequences of assuming regions with different diffusivities are demonstrated. The calculation was executed for 
$c_{0}=6 \times 10^{-5}$. To avoid instabilities in the numerical solution of equation (6) the time steps $\Delta t$ were adapted to the faster core diffusion process $\left(\Delta t=\Delta t_{\mathrm{C}}=5 \times 10^{-9} \mathrm{~s}\right)$.

Figure 3 shows the bowing-out distance $x_{\mathrm{d}}$ calculated in this way as a function of time. After a fast initial movement the dislocation slows down and $x_{\mathrm{d}}$ converges to a saturation value. This final limit is determined by the driving force $\left(F_{\sigma}-F_{\mathrm{L}}\right)$ and the force exerted by the "bulk solute" (i.e. solute atoms in the bulk region), which has not moved in the short time step. By comparison, the "core solute" (i.e. solute atoms in the core region) has moved with the dislocation and exerts only a negligible force. For simplicity, we will denote this regime of relaxation due to diffusion of solute in the core region as "core relaxation".

The balance of forces is illustrated in Fig. 4: the force $F_{\sigma}$ due to the external stress is constant, while the net driving force $F_{\sigma}-F_{\mathrm{L}}$ decreases linearly with increasing bowing-out distance. The back-driving force, which is exerted by the "bulk solute", is shown as the curved line $\left(F_{\mathrm{S}, \mathrm{B}}\right)$; after going through a maximum, $F_{\mathrm{S}, \mathrm{B}}$ approaches the driving force such that the velocity converges to zero and the bowing-out distance reaches a saturation value, as seen in Fig. 3 . Because of the asymptotic behavior, the calculation was stopped at

$$
0.975 \cdot F_{\sigma}-F_{\mathrm{L}}+F_{\mathrm{S}, \mathrm{B}}<0 .
$$

This corresponds to the end points marked in Figs 3 and 4.

As the bowing-out due to "core relaxation" saturates, further dislcoation motion requires diffusion of the "bulk solute". In this regime of "bulk relaxation" the time steps $\Delta t_{\mathrm{B}}$ were chosen to be larger by about a factor of $10^{6}\left(=D_{\mathrm{B}} / D_{\mathrm{C}}\right)$ than $\Delta t_{\mathrm{C}}$. To avoid instabilities in the core region the fact was used that after the first part of the calculation the solute atom distribution in the core region is nearly in equilibrium. Therefore, in the following calculation the

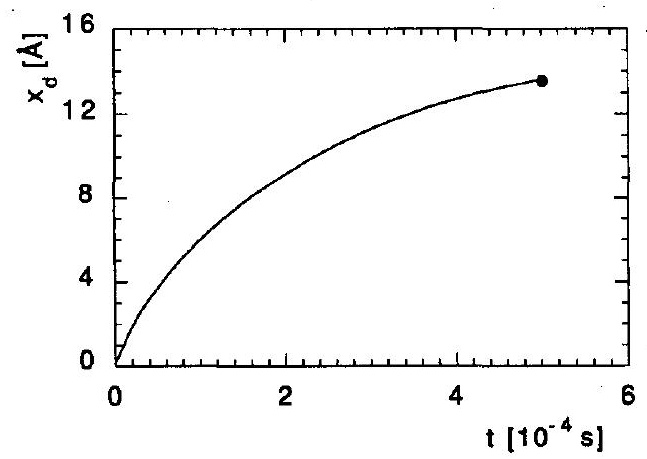

Fig. 3. The first stage of the bowing-out $\left(x_{\mathrm{d}, \mathrm{c}}\right)$ of a dislocation as a function of time. The kinetics of the movement is determined by fast diffusion of the "core solute". The calculation is interrupted at the end point of the curve.

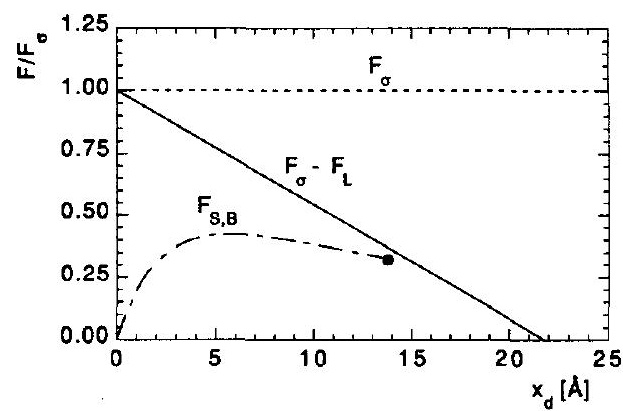

Fig. 4. The forces acting on the dislocation during the movement shown in Fig. 3 as a function of the bowing-out distance. The velocity in Fig. 3 falls as the net driving force $\left(F_{\sigma}-F_{\mathrm{L}}\right)$ approaches the back-driving force due to the "bulk solute" $\left(F_{\mathrm{S}, \mathrm{B}}\right)$. The bowing-out distance is determined by the force of the "bulk solute", which does not move within the time steps characteristic for diffusion in the dislocation core.

solute atom distribution in the core region was taken into equilibrium, without diffusion steps following equation (6) [15].

From these results the following conclusion concerning the role of the two solute regions, as shown schematically in Fig. 5, can be drawn: the kinetics of the first step (the "core relaxation regime") is controlled by the "core solute" [Fig. $5(\mathrm{a}-\mathrm{c})$ ]. However the maximum bowing-out distance, which is proportional to the relaxation strength, is determined by the "bulk solute". In the second step (the "bulk relaxation regime"), both the kinetics and the relaxation strength are determined by the diffusion of the "bulk solute" [Fig. 5(d,e)]. From the bowing-out distance in the "core and bulk relaxation" regimes as a function of time, the relaxation times and normalized strengths were calculated according to equations (8) and (9).

\subsection{The shape of the Cottrell cloud}

The shape of the Cottrell cloud, which is shown schematically in Fig. 5, was now calculated in the regimes of "core relaxation" and of "bulk relaxation". In the former case, $\sigma_{x y}=10^{-5} \cdot G, c_{0}=$ $3 \times 10^{-5}, l=500 \mathrm{~nm}$, and $T=400 \mathrm{~K}$ were chosen. The resulting Cottrell clouds for $x_{\mathrm{d}, \mathrm{C}}=0,3$ and $11 \AA$ are shown in Fig. 6(a-c), where the concentrations are plotted in the transformed $u, v$ space. The bowingout of the dislocation as a function of the time is plotted in Fig. 6(d). Figure 6(a-c) correspond to the schematic sketch in Fig. $5(a-c)$. The first plot [Figs 6(a) and 5(a)] shows the symmetric equilibrium distribution at the beginning. At an intermediate stage [Figs $6(\mathrm{~b})$ and $5(\mathrm{~b})$ ] the distributions of the "core solute" and of the "bulk solute" have become asymmetric. The strong forces from the "core solute" are reflected in the asymmetry of the Cottrell cloud near the dislocation. In the third plot [Figs 6(c) and $5(\mathrm{c})]$ the asymmetry of the core region has decreased, while the bulk region is heavily distorted. The conse- 

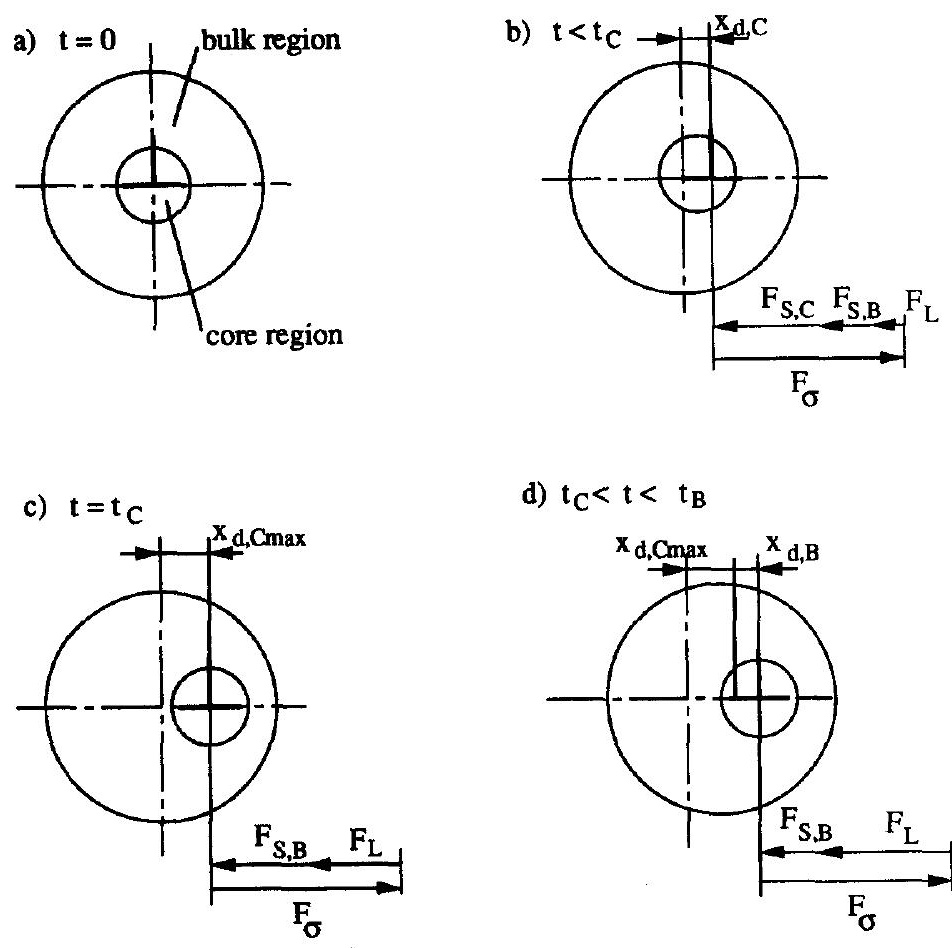

d) $\mathrm{t}_{\mathrm{C}}<\mathrm{t}<\mathrm{t}_{\mathrm{B}}$

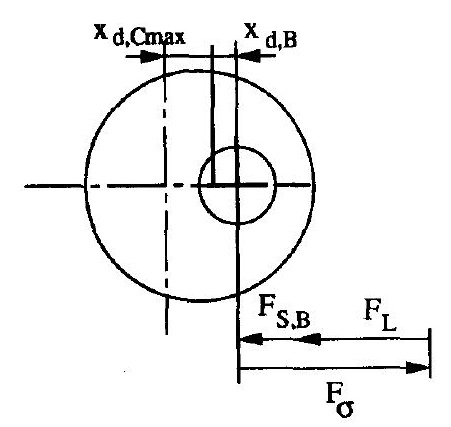

e) $t=t_{B} \gg t_{C}$

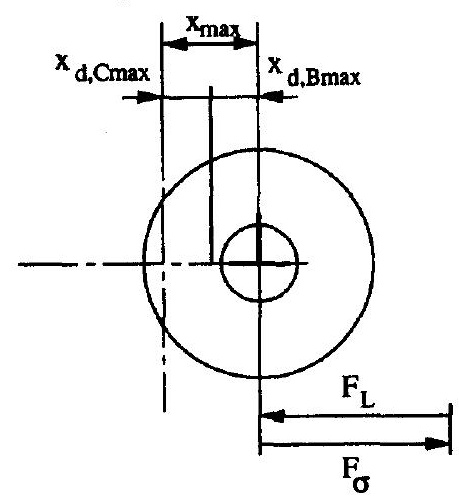

Fig. 5. Schematic of the sequence of events treated in the model: the shape of the Cottrell cloud in the core region (= small circle) and the bulk region (= large circle) is indicated for five different bowing-out distances. The forces on the dislocation (as in Fig. 4) are depicted for each stage, where $F_{\sigma}$ is the force due to the external stress, $F_{\mathrm{L}}$ the back driving force due to the line tension, $F_{\mathrm{S}, \mathrm{B}}$ the force exerted by the "bulk solute", and $F_{\mathrm{s}, \mathrm{c}}$ the force exerted by the "core solute". (a) Initial configuration: The solute clouds within the core and bulk region are symmetric. (b) Intermediate stage for "core relaxation": The "core solute" diffuses with the dislocation in contrast to the "bulk solute" which does not. The action of the forces $F_{\mathrm{S}, \mathrm{B}}$ and $F_{\mathrm{S}, \mathrm{C}}$ which balance the net driving force $\left(F_{s}-F_{\mathrm{L}}\right)$ is reflected in the asymmetry of the solute cloud. (c) "End point" configuration for the "core relaxation" and start configuration for the "bulk relaxation": at the end of the core relaxation the "core solute" distribution is nearly in equilibrium and the net driving force is balanced by the bulk solute. (d) Intermediate stage for "bulk relaxation": The "core solute" is in equilibrium while the bulk solute is still asymmetrically distributed. The external force is balanced by the force exerted by the bulk solute and the line tension. (e) "End-point" configuration for the "bulk relaxation": The solute distributions of the "core" and "bulk solute" are now in equilibrium.

The external force is balanced only by the line tension.

quence is a lower force of the "core solute" and a higher force of the "bulk solute" acting on the dislocation.

For calculating the solute distribution during " $b u l k$ relaxation", a higher solute concentration $c_{0}=$
$3 \times 10^{-4}$ was chosen. As a starting configuration, the "end-point" situation of the "core relaxation" was assumed. The evolution of the solute distribution in Fig. $7(\mathrm{a}-\mathrm{c})$ can be compared with the schematic in Fig. 5(c-e). It is seen that the asymmetry of 
the Cottrell cloud, and therefore the force $F_{\mathrm{S}, \mathrm{B}}$, decreases during the bowing-out. At the end of the calculation the solute distribution is nearly in equilibrium and the external force is balanced only by the line tension. The generally less-pronounced asymmetry in Fig. 7(a-c) when compared to Fig. 6(a-c) is due to the higher solute concentration.
3.3. The influence of the parameters $\sigma_{x y}, c_{0}, l$ and $T$

To study the effect of the solute concentration and of the external stress, parameters for which the dominating mechanism changes from "core" to "bulk relaxation" were selected. For studying the influence of $l$ and $T$, parameters were used for which one mechanism clearly dominates.

(a)

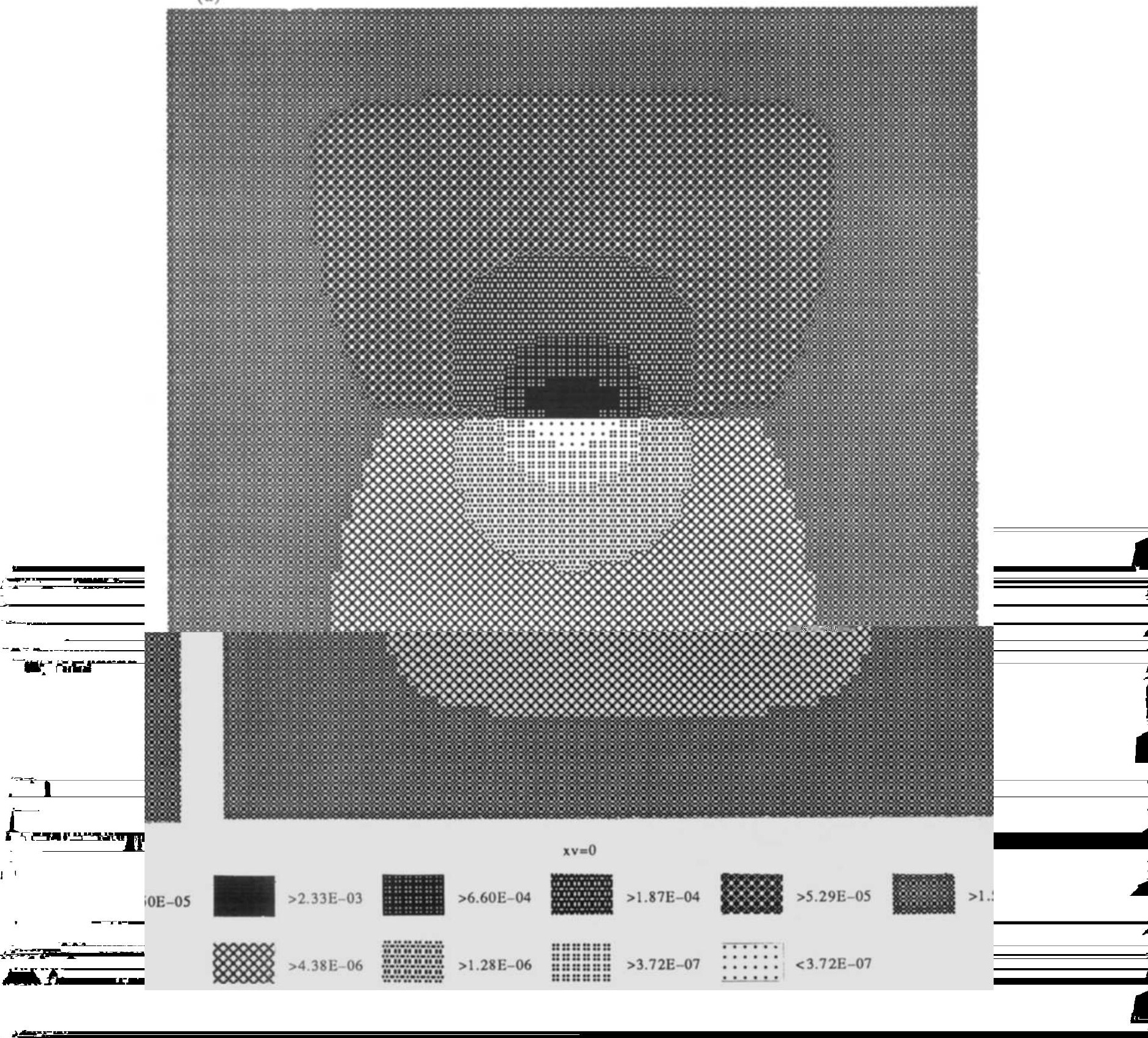


(b)

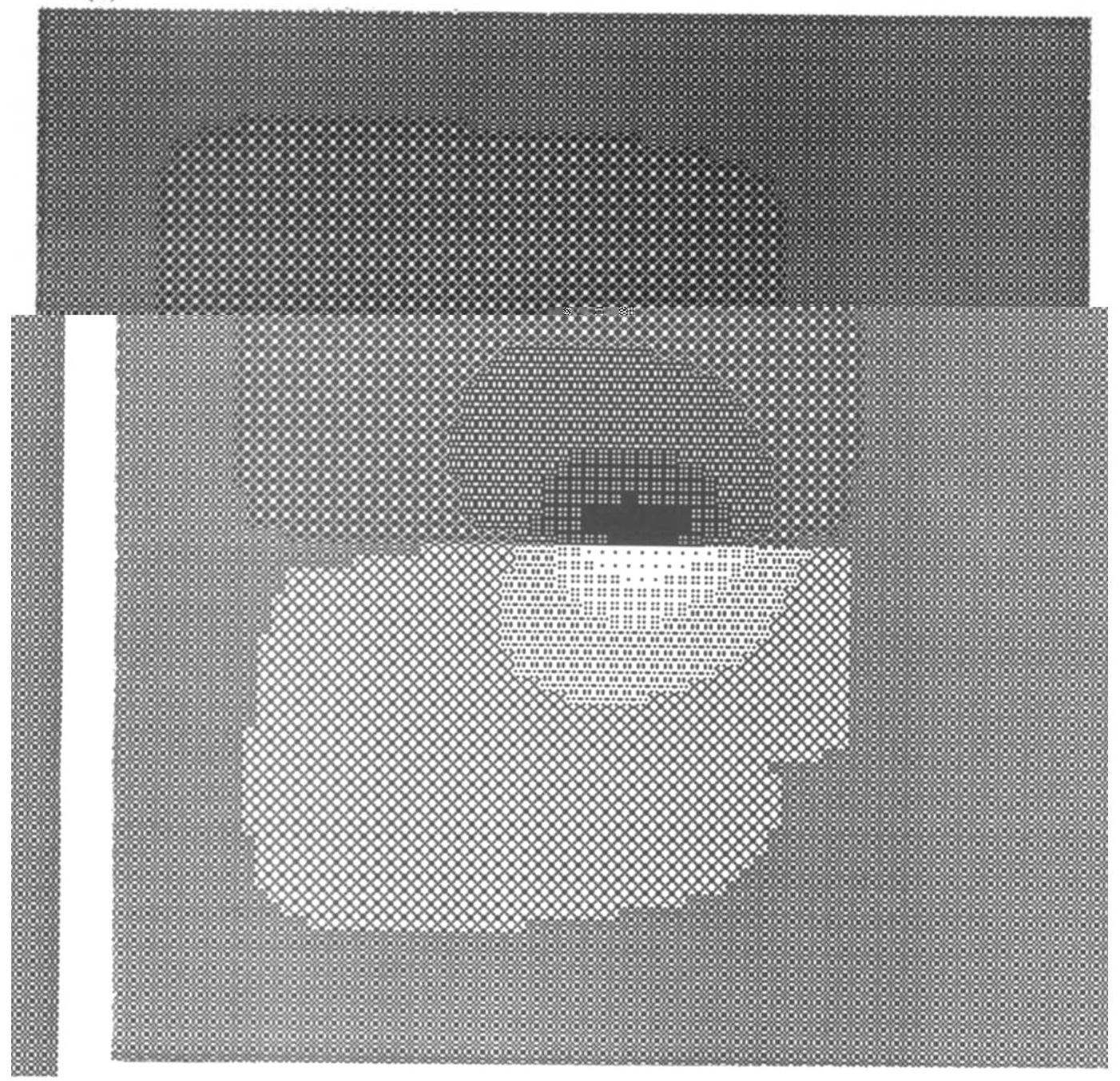

$50 \mathrm{E}-05$

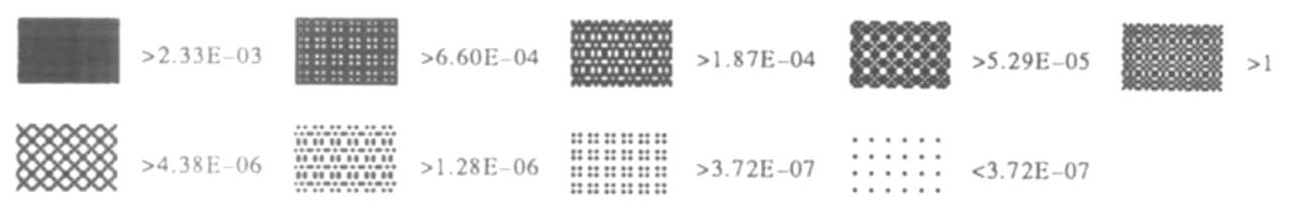

Fig. 6b. (Caption on previous page). 
(c)

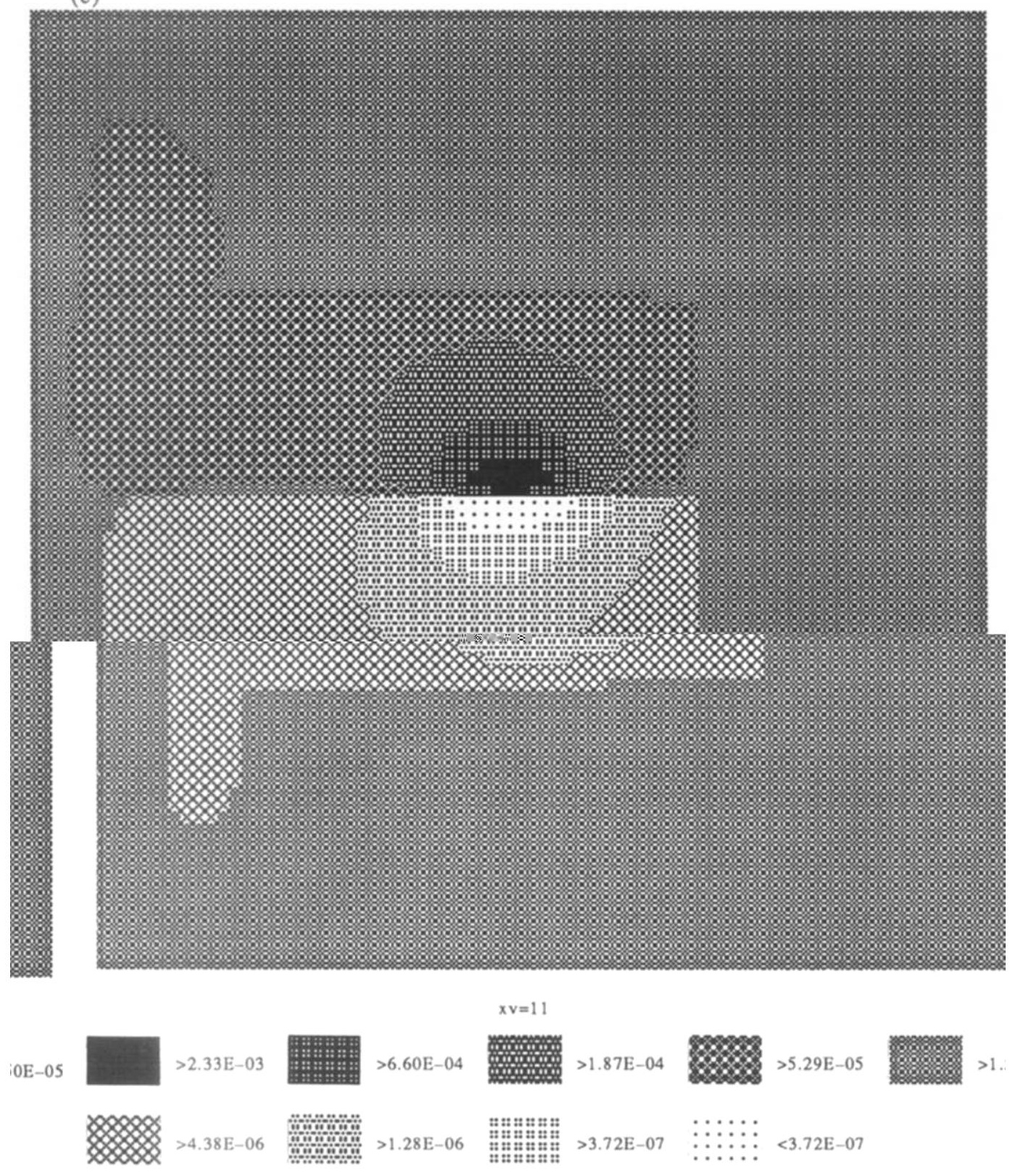


3.3.1. The influence of the solute concentration. In this investigation the solute concentration $c_{0}$ was varied between $2 \times 10^{-5}$ and $5 \times 10^{-4}$. In Fig. 8 the normalized relaxation strength and the relaxation time for the "core relaxation" are plotted as a function of the solute atom concentration. In Fig. 9 the same is plotted for the "bulk relaxation". For increasing concentration the relaxation strength for "core relaxation" decreases, whereas that for the "bulk relaxation" increases. This can be explained as follows: With rising concentration, the force due to the "bulk solute" grows and the driving force $\left(F_{\sigma}-F_{\mathrm{L}}\right)$ is therefore balanced at a shorter bowingout distance (see dash-dotted line in Fig. 4). The maximum bowing-out distance of the dislocation, which is the sum of the bowing-out in the "core" and "bulk relaxation" regime, is determined only by the line tension. Therefore a smaller bowing-out during "core relaxation" allows a larger bowing-out during "bulk relaxation" (and vice versa). It should be noted that, because of fitting errors in obtaining the relaxation strengths, the sum of $\Delta_{C}+\Delta_{B}$ is not exactly 1 .

(a)

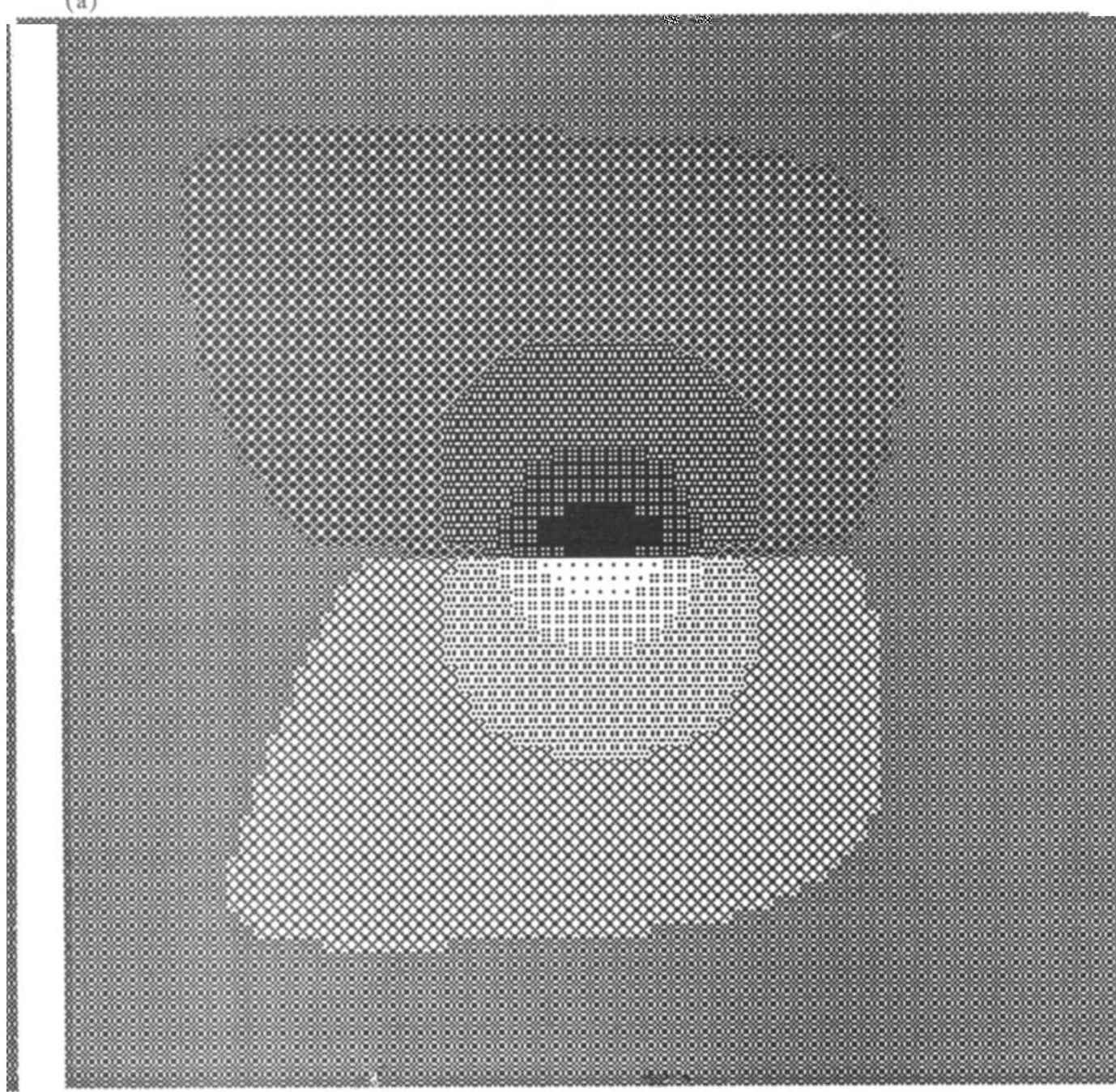

$\mathrm{xy}=2$

$-04$

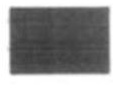

$>2.20 \mathrm{E}-02$

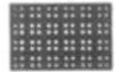

$>6.32 \mathrm{E}-03$

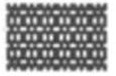

$>1.82 \mathrm{E}-03$

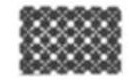

$>5.22 \mathrm{E}-04$

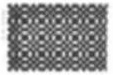

$>1.50 \mathrm{E}$

m

Fig. 7a

Fig. 7. Solute distribution as in Fig. 6, but for "bulk relaxation" $\left(c_{0}=3 \times 10^{-4}\right)$. The additional bowing-out distances after completion of the core relaxation are: (a) $x_{\mathrm{d}, \mathrm{B}}=2 \AA$; (b) $x_{\mathrm{d}, \mathrm{B}}=9 \AA$; (c) $x_{\mathrm{d}, \mathrm{B}}=19 \AA$; (d) Bowing-out distance as a function of the time. 
(b)

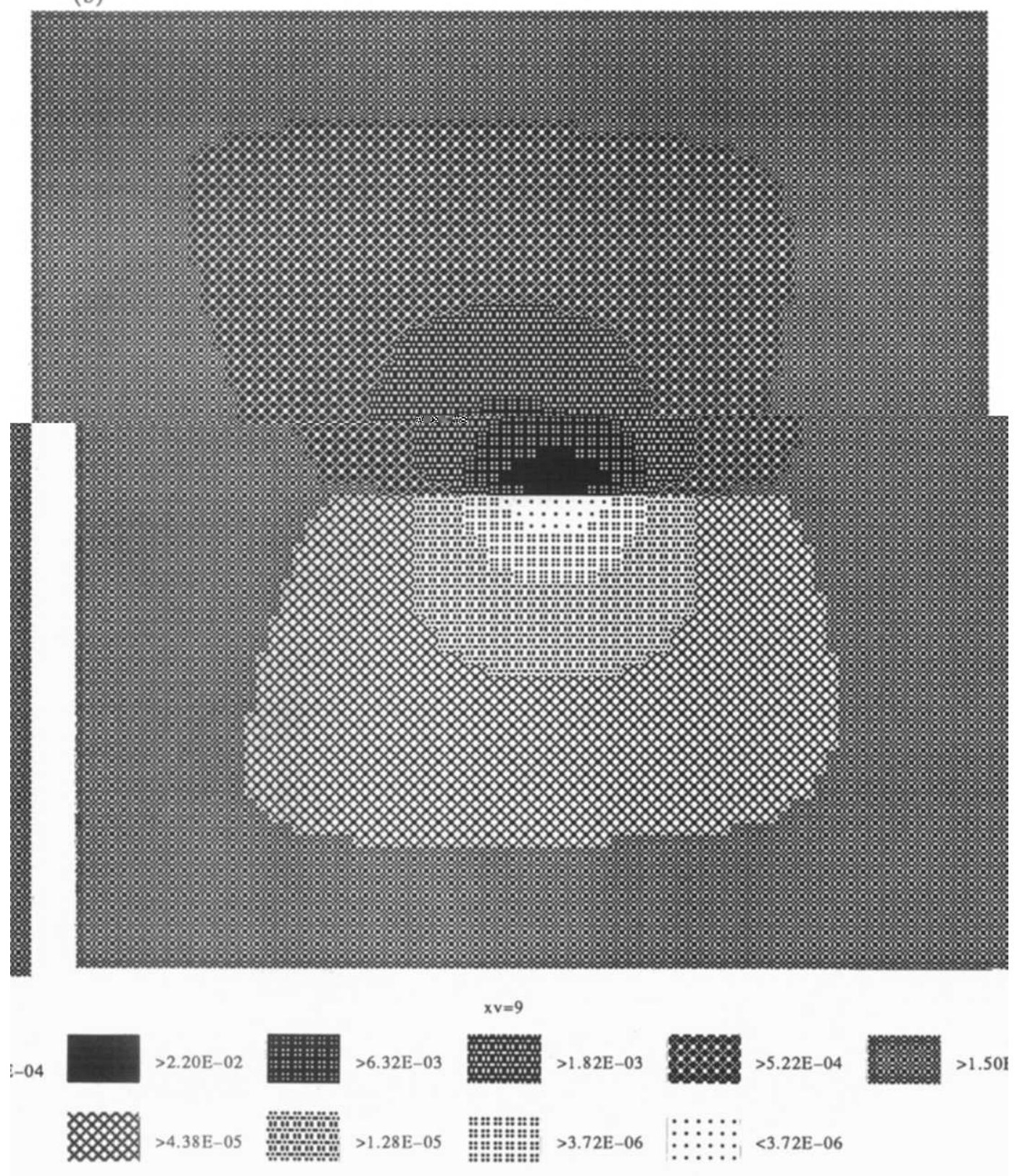

Fig. 7b. (Caption on p. 3793). 
(c)
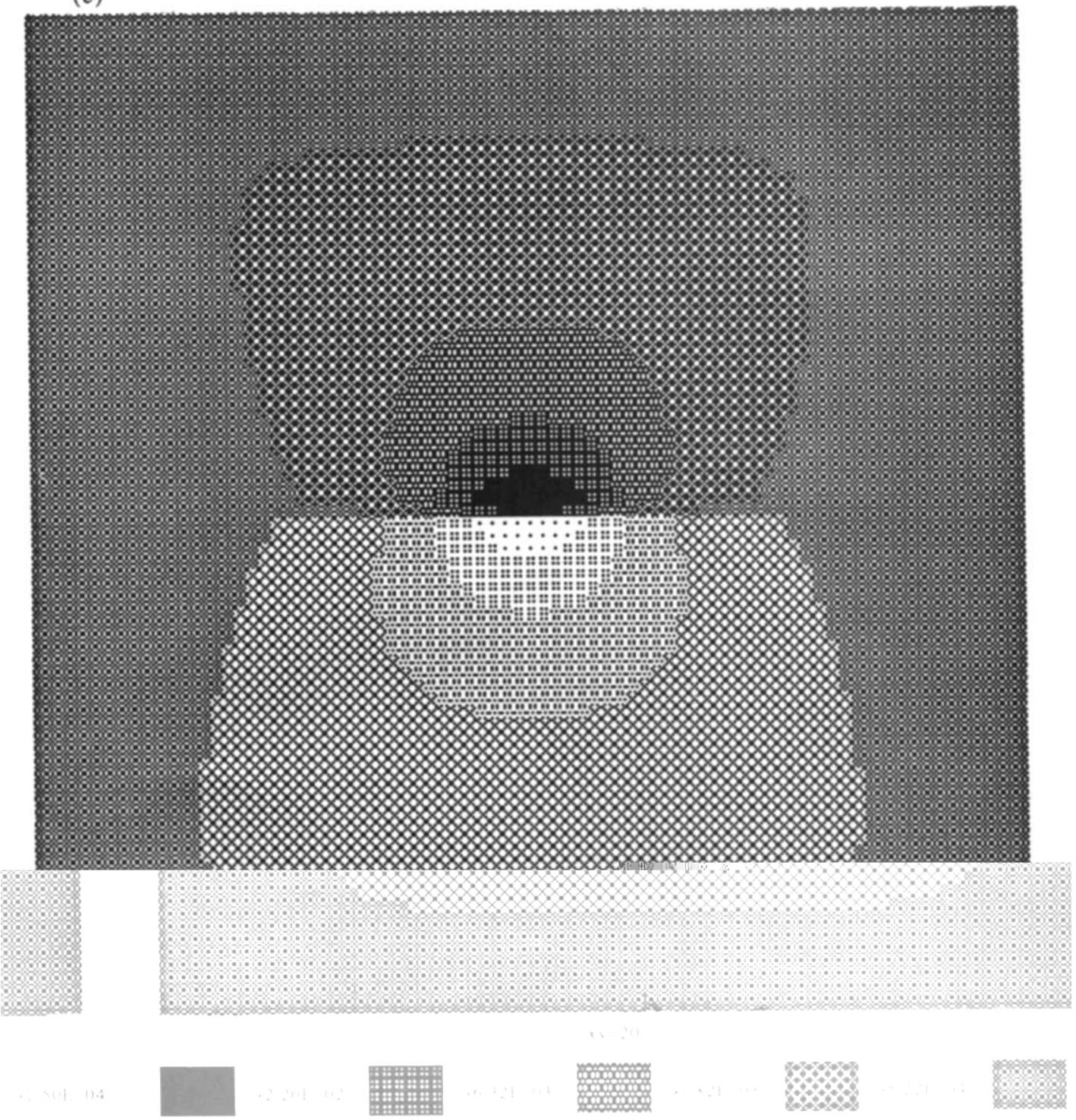

$m$

Fig. 7c. (Caption on p. 3793).

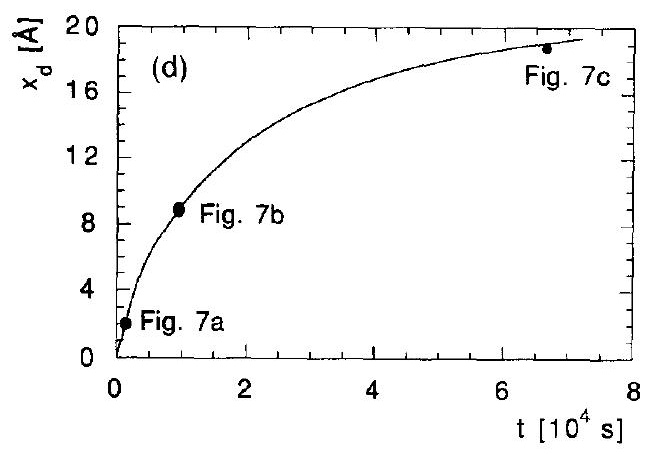

Fig. 7d. (Caption on p. 3793). 


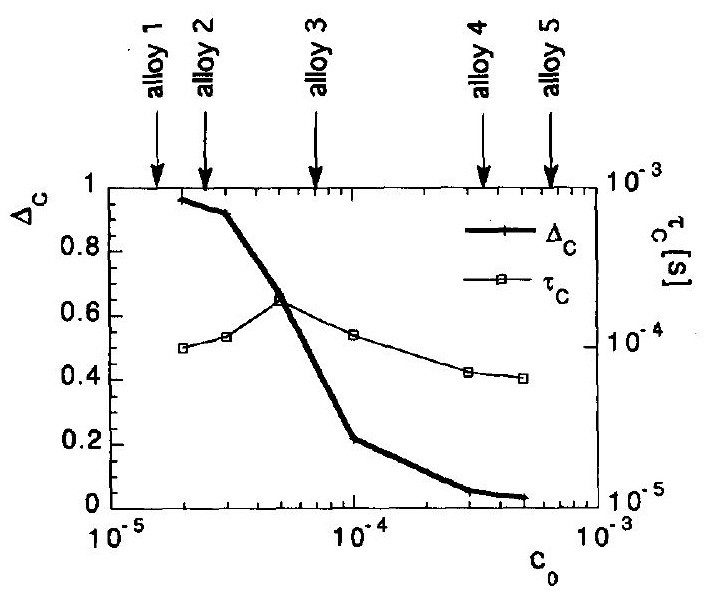

Fig. 8. The relaxation strength $\Delta_{\mathrm{C}}$ and the relaxation time $\tau_{\mathrm{C}}$ for "core relaxation" as a function of the solute atom concentration ( $\mathrm{Si}$ ) in the matrix (Al) for $T=400 \mathrm{~K}$, $\sigma_{x y}=G \cdot 10^{-5}$ and $l=500 \mathrm{~nm}$. The arrows correspond to the hypothetical alloys discussed with reference to Fig. 13.

The maximum in the "core relaxation" time can be explained by two opposite contributions. With rising solute concentration, the viscosity of the Cottrell cloud grows and an increasing relaxation time is expected. However, at higher concentrations the bowing distance decreases dramatically, which results, for the same velocity, in a shorter relaxation time. In the region of the maximum the viscosity of the cloud increases faster than the bowing-out distance falls. "Bulk relaxation", for which the bowing-out distance increases with $c_{0}$, does not show this effect and the relaxation time increases continuously.

3.3.2. The influence of the external stress. In Figs 10 and 11 the normalized relaxation strength and the relaxation time for "core relaxation" and "bulk relaxation" are plotted as a function of the external stress. The concentrations were fixed at $c_{0}=3 \times 10^{-5}$ (in the "core relaxation" regime) and $c_{0}=3 \times 10^{-4}$ (in the "bulk relaxation" regime). These figures reflect the fact that a falling external stress has the same influence on the relaxation strength as an increasing solute concentration. This can be explained with Fig. 4: a falling external stress shifts the curve $F_{\mathrm{S}, \mathrm{B}}$ to

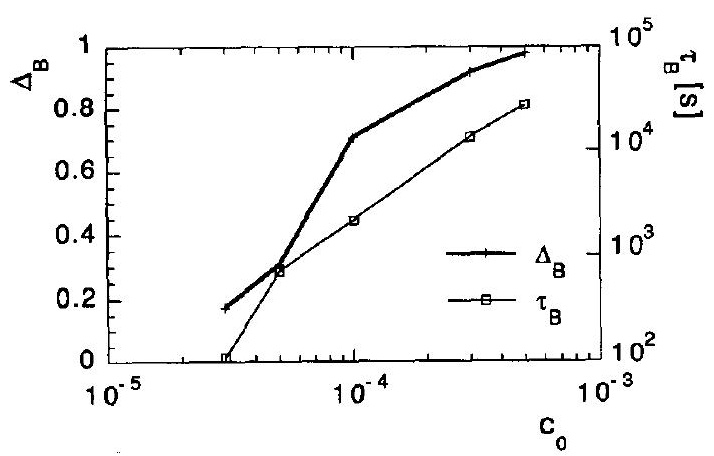

Fig. 9. Same as in Fig. 8 for "bulk relaxation".

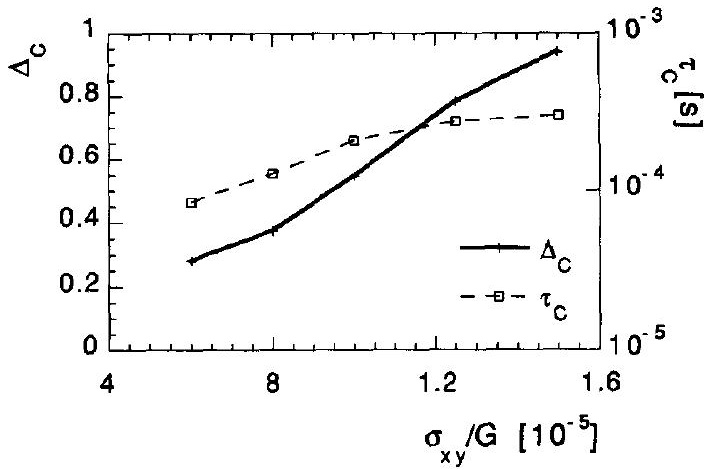

Fig. 10. The relaxation strength $\Delta_{C}$ and the relaxation time $\tau_{\mathrm{C}}$ for "core relaxation" as a function of the external stress for $T=400 \mathrm{~K}, c_{0}=3 \times 10^{-5}$ and $l=500 \mathrm{~nm}$.

higher values because of the normalization with $F_{\sigma}$; additionally, the line for the net driving force $\left(F_{\sigma}-F_{\mathrm{L}}\right)$ acquires a larger slope. Therefore, the bowing-out distance becomes shorter as in the case of increasing concentration in which only the curve $F_{\text {S.B }}$ is shifted to higher values.

3.3.3. The influence of the pinning length of the dislocation. The parameters $c_{0}=5 \times 10^{-5}$ for the "core relaxation" and $c_{0}=5 \times 10^{-4}$ for the "bulk relaxation" were kept constant. The pinning length $l$ of the dislocation was varied between 200 and $500 \mathrm{~nm}$. The dependencies of the relaxation strength and the relaxation time on $l$ turned out to follow power laws of the form $\tau \sim l^{n}$ and $\Delta \sim l^{m}$, where within the "core" and "bulk relaxation" regimes the exponents $n$ and $m$ are approx. 2. If the bowing-out of the dislocation is determined only by the line tension, the exponent is exactly 2 , as shown e.g. by Schoeck [18].

3.3.4. The influence of the temperature. The concentrations $c_{0}=5 \times 10^{-5}$ for the "core relaxation" and $c_{0}=5 \times 10^{-4}$ for the "bulk relaxation" were kept constant. From the calculated dependence of the relaxation time on the temperature the activation enthalpy can be determined according to $\tau=\tau_{0} \cdot \exp (H / k T)$, where $\tau_{0}$ is the inverse attempt frequency and $H$ the activation enthalpy of the

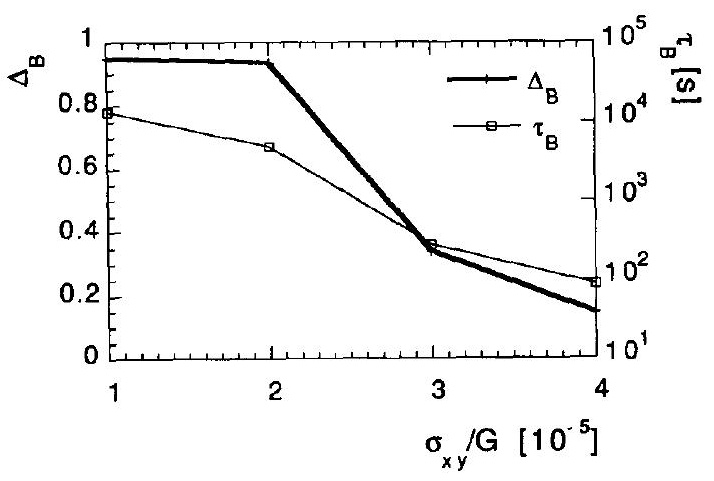

Fig. 11. Same as Fig. 10, but for "bulk relaxation" and $c_{0}=3 \times 10^{-4}$ 
relaxation process. For "core relaxation" we find

$$
\tau_{0}=3.7 \times 10^{-17} \mathrm{~s} \quad H=98 \mathrm{~kJ}
$$

and for "bulk relaxation"

$$
\tau_{0}=1.2 \times 10^{-15} \mathrm{~s} \quad H=147.8 \mathrm{~kJ} .
$$

It is remarkable that the values for the activation enthalpy turn out to be close to, but higher than, the activation enthalpies for diffusion $\left(Q_{\mathrm{D}, \mathrm{C}}=82 \mathrm{~kJ}\right.$ and $Q_{\text {D.B }}=142 \mathrm{~kJ}$ ). This additional temperature dependence can be explained as follows: with increasing temperature the Cottrell cloud "evaporates" and the lower viscosity results in shorter relaxation times. The difference between the measured activation enthalpy and the one for diffusion is thus a mean binding energy for solute atoms.

\section{DISCUSSION}

4.1. Comparison with analytical expressions on the basis of Schoeck's model

To allow an easier comparison between experimental data and the proposed model, analytical relations were derived on the basis of Schoeck's model $[6,18,27]$ and compared with the solutions of our numerical model. In Schoeck's model the relaxation time is expressed as

$$
\tau_{\mathrm{S}}=\frac{l^{2}}{5 \cdot G b^{2}} \cdot \frac{k T}{D \cdot \Delta l}
$$

where $D$ is the solute diffusivity and $\Delta l$ the mean distance between solute atoms along the dislocation.

In the case of "core relaxation", the appropriate value for $\Delta l$ is the mean distance between the solute atoms in the dislocation core. The concentration is estimated by the Boltzmann distribution $c_{\mathrm{C}}=$ $c_{0} \cdot \exp \left(U_{\mathrm{B}} / k T\right)$, where for the binding energy $U_{\mathrm{B}}$ the value of the interaction energy [equation (3)] at $x, y=0$ is used. Therefore, the relaxation time can be expressed as

$$
\tau_{\mathrm{S}, \mathrm{C}}=\frac{l^{2}}{5 \cdot G b^{2}} \cdot \frac{k T}{D} \cdot \frac{c_{0}}{b} \cdot \exp \left(U_{\mathrm{B}} / k T\right)
$$

A calculation of the relaxation time yields (for $l=500 \mathrm{~nm}, c_{0}=5 \times 10^{-5}, U_{\mathrm{B}}=0.23 \mathrm{eV}$ )

$$
\tau_{\mathrm{S}, \mathrm{C}}=1.6 \times 10^{-16} \cdot \exp (104,200 / R T) \mathrm{s}
$$

in comparison to

$$
\tau_{\mathrm{C}}=4 \times 10^{-17} \cdot \exp (98,000 / R T) \mathrm{s}
$$

for our model. The discrepancy in the activation enthalpy can probably be explained by the constant binding energy assumed in Schoeck's model as opposed to the variable binding energy $E=E(x, y)$ in our model.
In the case of "bulk relaxation", $\Delta l$ is identified with the mean distance between the solute atoms in a hollow cylinder with cut-off radii $r_{\mathrm{i}}$ and $r_{\mathrm{a}}$. The result is (Appendix 2)

$$
\tau_{\mathrm{S}, \mathrm{B}}=\frac{l^{2}}{5 \cdot G b^{2}} \cdot \frac{k T}{D} \cdot \frac{\pi \cdot A^{2}}{k^{2} T^{2}} \cdot \frac{c_{0}}{\Omega} \cdot \frac{1}{2} \cdot \ln \left(\frac{r_{\mathrm{a}}}{r_{\mathrm{i}}}\right) .
$$

For $r_{\mathrm{i}}=b, r_{\mathrm{a}}=10 b, l=500 \mathrm{~nm}, c_{0}=5 \times 10^{-4}$ and the parameters in Table 1 we find

$$
\tau_{\mathrm{S}, \mathrm{B}}=3.2 \times 10^{-14} \cdot \exp (142,000 / R T) \mathrm{s}
$$

in comparison to

$$
\tau_{\mathrm{B}}=1.2 \times 10^{-15} \cdot \exp (147,400 / R T) \mathrm{s}
$$

for our model. The differences in the inverse attempt frequency and activation enthalpy arise from the fact that a binding energy was not considered in the approximation in Appendix 2.

In Schoeck's model the relaxation time is proportional to the solute atom concentration. As shown in Section 3.3, this is only correct for large concentrations, at which only "bulk relaxation" is possible. For "core relaxation" there are always deviations because the maximum bowing-out is influenced by the force of the "bulk solute". In spite of this discrepancy the analytical equation may be used for comparison with the experimental results if the dominant mechanism is known.

\subsection{A mechanism map and consequences for the internal friction spectrum}

The new feature in the present numerical model for internal friction due to solute drag by dislocations is the consideration of a region near the dislocation core with a higher solute mobility normal to the dislocation. This model has yielded two relaxation steps: the kinetics of the first one is determined by fast diffusion of the solute atoms near the dislocation core, whereas the kinetics of the second one is controlled by the solute far away from the dislocation core. The relaxation strengths of the two processes depend strongly on the solute concentration, the external stress and the elastic misfit of the solute atoms. The parameters $l$ and $T$ by contrast, have only a slight influence and are not considered in the following. Therefore, for a given alloy a "map" with the axes external stress and solute concentration can be calculated in which the domains for the "core" and "bulk relaxation" are delineated. At the boundary between the domains the relaxation strengths for the two mechanism are equal. Such a map is shown for Al with $\mathrm{Si}$ solute atoms in Fig. 12. The values for this map originate from evaluations of Figs 8-11 and similar calculations, and an approximate method [15].

The position of a given alloy on this map will determine its internal friction spectrum in the following way. An alloy with a very low solute concen- 


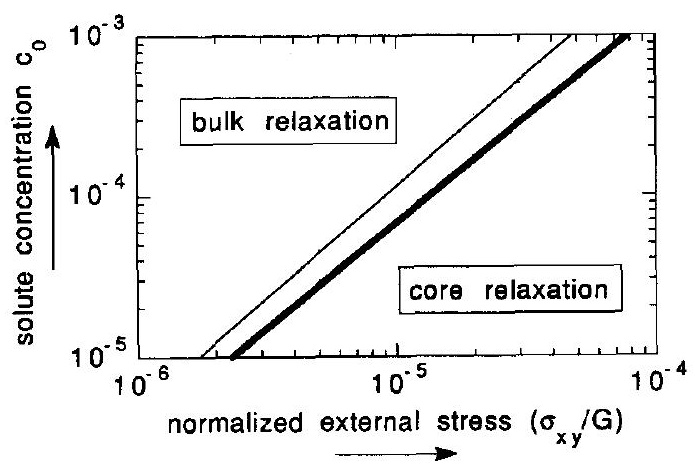

Fig. 12. Mechanism map for $\mathrm{Al}-\mathrm{Si}$, which shows the dominant relaxation mechanism as a function of the external stress $\sigma_{x y}$ and the solute concentration $c_{0}$ for $T=400 \mathrm{~K}$ and $l=500 \mathrm{~nm}$. The boundaries are calculated with the model described in Section 2 (heavy line) and the approximation method in [15].

tration (hypothetical alloy 1 in Fig. 13) clearly lies in the "core relaxation" domain, which implies that the anelastic bowing is controlled by diffusion of the "core solute" (compare also Fig. 8). Therefore a large low temperature maximum and a negligible high temperature maximum would be expected. The activation enthalpy will be the sum of the activation enthalpy for diffusion and the binding energy between solute atoms and the dislocation. For the small

(a)

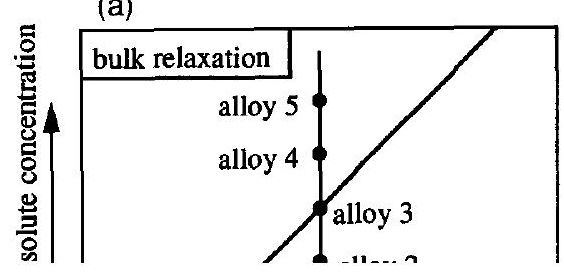

maximum, due to "bulk relaxation", the kinetics is determined by the diffusion of the "bulk solute" and the activation enthalpy for the relaxation process contains mainly this term. If the solute concentration is raised from alloy 1 to alloy 5 (Fig. 13), "bulk relaxation" is expected to dominate increasingly. Therefore the high temperature maximum in the internal friction spectrum will be enlarged at the expense of the low temperature maximum. If the external stress is varied from high to low values, a similar sequence of internal friction spectra will result.

It is encouraging that several experimental studies seem to be in agreement with these predictions of our model. For example, early results on $\mathrm{Cu}-\mathrm{Si}$ and $\mathrm{Cu}-\mathrm{Al}$ alloys with different solute concentrations [28] follow the sequence as schematically shown in Fig. 13(b). The calculated crossover from "core" to "bulk relaxation" is even in the same order of magnitude as in the experimental [29]. Our own damping studies on a single crystal $\mathrm{Al}-\mathrm{Si}$ alloy revealed a maximum at $450 \mathrm{~K}(f \sim 4 \mathrm{~Hz})$, which had to be attributed to dislocation motion $[15,16]$. By contrast, the maximum occurs at a significantly higher temperature in an $\mathrm{Al}-\mathrm{Mg}$ [30] alloy with a higher solubility. Again, a change from "core" to "bulk relaxation" is a likely explanation for the displacement of the maximum. A detailed discussion of new and published results in the light of our model will be given in the companion paper [29].

\section{CONCLUSION}

A model for explaining internal friction (mechanical loss) due to dislocations which interact with substitutional solute atoms is proposed. It is assumed cos o 


\section{REFERENCES}

1. G. Fantozzi, C. Esnouf, W. Benoit and I. G. Ritchie, in Progress in Materials Science, Vol. 27 (edited by J. W. Christian, P. Haasen and T. B. Massalski), p. 311. Pergamon Press, New York (1983).

2. A. Seeger, J. Physique 42, C5-201 (1981).

3. K. Lücke and A. V. Granato, Phys. Rev. B. 24, 6991, 7007 (1981).

4. I. G. Ritchie, Scripta metall. 16, 249 (1982).

5. M. L. Nó, C. Esnouf, J. San Juan and G. Fantozzi, Acta metall. 36, 827, 837 (1988)

6. G. Schoeck, Acta metall. 11, 617 (1963)

7. A. Seeger, Physica status solidi (a) 55, 457 (1979).

8. A. V. Granato and K. Lücke, J. appl. Phys. 52, 7136 (1981).

9. J. S. Koehler, in Imperfections in Nearly Perfect Crystals (edited by W. Shockley, J. H. Hollman and R. Maurer), p. 197. Wiley, New York (1952).

10. A. Granato and K. Lücke, J. appl. Phys. 27, 583 (1956).

11. K. Lücke and J. Schlipf, in The Interaction between Dislocations and Point Defects, Vol. I (edited by B. L. Eyre), AERE Rep. 5944, Harwell, p. 118 (1968).

12. D. G. Blair, T. S. Hutchinson and D. H. Rogers, Can. J. Phys. 49, 633 (1971).

13. W. Winkler-Gniewek, J. Schlipf and R. Schindlmayr, Proc. ICIFUAS 5 (edited by D. Lenz and K. Lücke), p. II-246 Springer, Berlin (1975).

14. W. Winkler-Gniewek, Doctoral dissertation, Aachen (1973).

15. A. Pichler, Doctoral dissertation, Univ. Leoben and Max-Planck-Institut für Metallforschung, Stuttgart (1992).

16. A. Pichler, M. Weller, E. Arzt and J. Diehl, Mater. Sci. Forum 119-121, 365 (1993).

17. K. Yamafuji and C. L. Bauer, J. appl. Phys. 36, 3282 (1956).

18. G. Schoeck, Scripta metall. 16, 233 (1982).

19. J. P. Hirth and J. Lothe, Theory of Dislocations, 2nd edn, p. 218. Wiley, New York (1982)

20. P. N. Beshers, Acta metall. 6, 521 (1958).

21. D. M. Barnett, G. Wong and W. D. Nix, Acta metall. 30, 2035 (1982).

22. M. Yoshinaga and S. Morozumi, Phil. Mag. 23, 1367 (1971).

23. M. Sakamoto, Mater. Trans. JIM 30, 337 (1989).

24. W. James and D. M. Barnett, in Solute Defect Interaction-Theory and Experiment (edited by $\mathbf{S}$. Saimoto and G. R. Purdy), p. 136. Pergamon Press, Toronto (1986).

25. J. L. Murray and A. J. McAlister, Bull. Alloy Phase Diagrams 5, 74 (1984)

26. H. J. Frost and M. F. Ashby, Deformation Mechanismen Maps, p. 21. Pergamon Press, Oxford (1982).

27. G. Schoeck, Scripta metall. 22, 389 (1988).

28. S. Weinig and E. S. Machlin, Trans. Am. Inst. Min. Engrs 209, 32 (1957).

29. A. Pichler, M. Weller and E. Arzt, Acta metall. mater. 42, 3801 (1994)

30. S. P. Wu and L. D. Zhang, Mater Sci. Engng $A$ 132, 225 (1991).

31. J. P. Hirth and J. Lothe, Theory of Dislocations, 2nd edn, p. 506. Wiley, New York (1982).

\section{APPENDICES}

\section{A1. The Back Stress due to Bowing-out of the Dislocation}

A shear stress $\sigma_{x y}$ produces a force $\sigma_{x y} \cdot b$ on a dislocation, and the resulting curvature of a pinned dislocation is given by

$$
\sigma_{x y} \cdot b=\frac{T_{\mathrm{L}}}{R} \sim \frac{G b^{2}}{2 R} .
$$

For constant line tension the pinned dislocation forms a circular arc, which for small bowing-out results in

$$
\sigma_{x y} \cdot b=4 \cdot \frac{G \cdot b^{2}}{l^{2}} \cdot x_{\mathrm{d}} \text {. }
$$

As this stress is necessary for bowing-out a dislocation to $x_{d}$, $\sigma_{x y}$ may be viewed as a back stress $\left(\sigma_{\mathrm{L}}\right)$ which diminishes the external stress.

\section{A2. The Relaxation Time for the "Bulk Relaxation"}

For calculating $\Delta l$ and $\tau$ for "bulk relaxation" the fundamental assumption is that the solute atoms in core region move so easily that they have no influence on the movement of the dislocation. As the calculation extends only across the bulk region, the concentration distribution may be computed from the stress field of a normal dislocation and Boltzmann statistics

$$
c=c_{0} \cdot \exp \left(-\frac{A \cdot \sin \Theta}{r \cdot k T}\right)
$$

where $\Theta$ and $r$ are the polar coordinates of the solute atoms. The number of additionally segregated atoms is given by [31]

$$
N=L \int_{r_{\mathrm{i}}}^{r_{\mathrm{a}}} r \cdot \mathrm{d} r \int_{0}^{2 \pi} \frac{c-c_{0}}{\Omega} \mathrm{d} \Theta
$$

where $L$ is the length of the dislocation segment, $r_{i}$ is an inner and $r_{2}$ an outer cutoff radius. The integrals are evaluated by expansion to a power series and term-wise integration [31]. If only the first term of the series and Schoeck's definition of $\Delta l$ is used, the equation for the relaxation time becomes [15]

$$
\tau_{\mathrm{S}, \mathrm{B}}=\frac{l^{2}}{5 \cdot G b^{2}} \cdot \frac{k T}{D} \cdot \frac{\pi \cdot A^{2}}{k^{2} T^{2}} \cdot \frac{c_{0}}{\Omega} \cdot \frac{1}{2} \cdot \ln \left(\frac{r_{\mathrm{a}}}{r_{i}}\right) .
$$

\section{NOMENCLATURE}

$b \quad$ Burger's vector

$c(x, y) \quad$ solute concentration field

$c_{0} \quad$ solute concentration far away from the dislocation

$l \quad$ pinning length of the dislocation

$\Delta l \quad$ mean distance between solute atoms along the dislocation

Boltzmann's constant

$k \quad$ Boltz

$\Delta t_{\mathrm{B}}, \Delta t_{\mathrm{C}} \quad$ time step for "bulk relaxation" and "core relaxation", resp.

$x_{\mathrm{d}} \quad$ bowing-out distance

$x_{\mathrm{d}, \mathrm{B}}, x_{\mathrm{d}, \mathrm{C}}$ bowing-out distance for "core" and "bulk relaxation", resp.

$D \quad$ diffusivity

$F_{\mathrm{S}, \mathrm{B}}, F_{\mathrm{S}, \mathrm{C}} \quad$ force per unit length due the solute atoms in the bulk region and solute atoms in the core region, resp.

$F_{\sigma}, F_{\mathrm{L}} \quad$ force per unit length due to the external stress and line tension, resp.

$G \quad$ shear modulus

$H \quad$ activation enthalpy

$T \quad$ absolute temperature

$T_{\mathrm{L}} \quad$ line tension of the dislocation

$U_{\mathrm{B}} \quad$ binding energy between a solute atom and a dislocation 
$\sigma_{\mathrm{L}} \quad$ back stress due to the line tension

$\sigma_{x y} \quad$ external shear stress

$\tau_{0} \quad$ inverse attempt frequency

$\tau_{\mathrm{B}}, \tau_{\mathrm{C}} \quad$ relaxation time for the "bulk relaxation" and
$\tau_{\mathrm{S}, \mathrm{B}}, \tau_{\mathrm{S}, \mathrm{C}} \quad$ relaxation time after Schoeck's model for the "bulk" and "core relaxation", resp.

$\Delta_{B}, \Delta_{C}$ relaxation strength for the "bulk" and "core relaxation", resp. 